\title{
EDITORIAL
}

\section{The Neuropsychology of Schizophrenia Circa 2009}

\author{
Robert M. Bilder
}

Received: 24 July 2009 /Accepted: 29 July 2009 / Published online: 13 August 2009

(C) The Author(s) 2009. This article is published with open access at Springerlink.com

Keywords Neuropsychology - Cognitive neuroscience . Genetics · Informatics · Neuroimaging · Phenomics .

Schizophrenia

The last 30 years have been marked by the emergence of transformative technologies for the study of brain structurefunction relations, and these have been deployed vigorously to help unravel the mysterious causes for, and treatments for the schizophrenia syndrome. Despite the progress, the ultimate goal - to identify a "smoking gun," in the form of a cognitive, a functional anatomical or a genetic signature responsible for the brain pathology underlying schizophrenia-remains elusive. This collection of articles from global leaders in neuropsychological research on schizophrenia makes poignant how much our thinking has changed over the last three decades, but also that we still have more questions than answers about the fundamental neurobiological underpinnings of schizophrenia.

To put the progress in perspective, we may recall the conclusions of Heaton et al. (1978), following their incisive review of neuropsychological studies of psychiatric disorders:

"The finding that chronic or process schizophrenics look like organics on neuropsychological tests might

R. M. Bilder $(\bowtie)$

Department of Psychiatry \& Biobehavioral Sciences,

David Geffen School of Medicine, Department of Psychology,

College of Letters and Science, and Semel Institute for

Neuroscience \& Human Behavior, UCLA,

Los Angeles, CA, USA

e-mail: rbilder@mednet.ucla.edu be considered surprising ... One fairly popular explanation $\ldots$ is that ... motivational deficiencies and thought disorders are responsible ... the implication is that these deficits are functional in nature. This explanation cannot be ruled out on the basis of currently available evidence, but it can be questioned" (page 156); and "[it is likely that] chronic schizophrenics will appear organic on neuropsychological testing because a significant proportion of them are organic" (page 157).

These prescient (and diplomatic) statements signal what was then a prevalent dualistic perspective, the echoes of which are now only faint. For example, one may note that the term "organic" was effectively scrubbed from the DSM-IV, contrasting to its conspicuous presence in the DSM-III. If the 21 st century is one day seen as marking the death of dualism in psychiatric taxonomies, this may be considered a victory for neuropsychology, neuroimaging (which provided compelling evidence of structural brain anomalies (Johnstone et al. 1976)), and genetics (confirming heritability of $\sim 80 \%$ for the schizophrenia syndrome). Whatever the driving forces, it is now commonplace to seek biological explanations for both the causes and treatments of schizophrenia, and this reflects a sea change in thinking for which neuropsychology can claim significant credit.

The reviews in this issue offer snapshots from multiple perspectives embraced in 21 st century research on the neuropsychology of schizophrenia. These papers show that neuropsychology has become central to the study of both the causes and treatments of schizophrenia.

- Palmer, Dawes, and Heaton offer a broad historical view, highlighting the role neuropsychological research 
has played in crystallizing modern thinking about schizophrenia. These studies have generated solid consensus about the severity, pattern, and typical course of deficits. Perhaps most important has been appreciation of the impact of these deficits on outcomes, leading to the view that cognitive impairments are indeed more important than the symptoms now used to diagnose the disorder, both to advance understanding of pathophysiology and to target for effective treatment.

- Corresponding to the increased appreciation that cognitive deficits are central to schizophrenia, research on neuropsychological consequences of treatment has expanded dramatically. Harvey provides an incisive survey of psychopharmacological strategies that have been tried so far, and some that remain on the horizon. Following what might be described as excessive (if not "irrational") exuberance over the cognitive gains seen in early studies of the "atypical" antipsychotics, steady progress has been made laying the groundwork for systematic efforts to both identify agents capable of modifying cognition in schizophrenia, and facilitate investigation of these agents using consensus methods (e.g., as accomplished by the MATRICS and TURNS initiatives).

- Stimulated in part by disappointment over the lack of efficacy and effectiveness of new psychopharmacological treatments, the last decade also has witnessed a surge of interest in non-pharmacological strategies to treat cognitive deficits in schizophrenia. Medalia and Choi provide an important perspective on the maturation of cognitive remediation research in schizophrenia, highlighting what are now appreciated as consistent moderate effects (which are greater than observed for any drug treatments so far), and emerging consensus regarding the critical mediators and moderators of these treatments.

- One clear mark of the maturation of neuropsychological research on schizophrenia is the increased emphasis on identification of its developmental precursors, with an eye towards development of early intervention or even prevention strategies. Two unique perspectives on these issues are provided here (Pantelis et al.; Niendam et al.). Pantelis et al. survey the results of neuropsychological and neuroimaging findings in individuals at high risk for later development of schizophrenia, and while there are promising leads, they conclude that further work focused on the dynamic, longitudinal patterns of change in these indicators may be necessary going forward. Niendam et al. offer a compatible perspective, but highlight the critical importance of understanding both social outcomes, and social cognitive predictors of these outcomes, to gain a stronger appreciation of the prodromal state and to aid in the design of optimal intervention or prevention strategies.

- Palmer et al. note that despite progress, there remains no consensus about the fundamental pathophysiological processes involved in schizophrenia at either neural systems or cognitive levels. Three integrative reviews illustrate current thinking about these grand challenges. Starting from the rational premise that episodic memory deficits have emerged as the most consistent and robust markers of schizophrenia, Leavitt and Goldberg critically evaluate the relevant neuroanatomical, genetic, and behavioral evidence. They conclude that integrative strategies, including advanced computational modeling together with functional neuroimaging, may help advance clearer mechanistic models of the memory dysfunction that is already a well validated marker of disability. Krauss, Keefe, and Krishnan present a highly innovative perspective that aims to offer a unified theory through which a common mechanism, based on memory prediction errors, may help understand not only why "memory deficits" are so prominent, but also why people with schizophrenia suffer from a range of other symptoms, including disorders of perceptual processing. Such a perspective might help significantly reorient thinking about the memory failures of schizophrenia, and other signs, with respect to specific deficits in neuronal architecture, and more specific basic cognitive mechanisms underlying anomalies in extracting and utilizing regularities of prior experience. Finally, the contribution of Gold, Hahn, Strauss \& Waltz really does aim to "turn it upside down" and ask what we can learn from what is not impaired in people with schizophrenia. While some might see the search for the holy grail of a specific deficit in schizophrenia as having met with abject failure, and adopted instead a generalized deficit model, there are clearly areas of cognitive performance that may, by virtue of their preservation, point us towards both clearer understanding of pathological processes (in part by ruling out certain possibilities), and further highlight potential targets for remediation based on preserved functions.

While these studies provide an enormous breadth and depth of coverage, no collection of papers can reflect the complete spectrum of current thinking about the neuropsychology of schizophrenia nor predict well what the "next big thing" may be. Recent genome-wide association studies have highlighted what is likely to be greater complexity of schizophrenia genetics than was previously anticipated, 
with thousands of common alleles each having small contributions to risk for both schizophrenia and bipolar disorder (The International Schizophrenia Consortium 2009). It is now also clearer that a broad collection of "generalist" genes likely explain the heritability of diverse cognitive traits (Butcher et al. 2006). These observations suggest that neuropsychology research is primed to move beyond the custom of studying cognitive domains that were originally validated with respect to discrete brain lesions, and begin to redefine neuropsychological constructs with respect to distributed neural systems functions, cellular systems and signaling pathways, and molecular variations (Bilder et al. 2009b, c; Sabb et al. 2009).

Neuropsychology research and practice are further poised to capitalize on the revolution in information technology. Not long ago it would be reasonable to believe that the "wisdom of crowds" was an obvious oxymoron. But the success of Wikipedia and other social collaborative networking applications have made it clear that large numbers of individuals can generate novel intellectual products with high utility. Some applications already enable collaborative knowledge-base development for cognitive phenotypes, linking hypotheses about cognitive concepts to the empirical data on which these hypotheses are based (Bilder et al. 2009a, b, c; Sabb et al. 2008), and a new Society for Neuroinformatics in Neuropsychology has just been established (see http://www.scnn.org/). There is further hope that similar efforts can bear fruit for clinical neuropsychology, by establishing collaborative databases and developing open-access assessment instruments. These strategies may one day overcome some of the limitations of laboratory- and clinic-based methods, and enable knowledge to be accumulated from much larger communities, substantially complementing and extending current methods. Working together with patient-oriented networks, there is hope that in another three decades, a special issue on the neuropsychology of schizophrenia will have answers to many of our current questions, and that previously unimaginable enigmata will have replaced those that we confront today.

Acknowledgements Supported by the NIH Roadmap for Medical Research/Common Fund, including Consortium for Neuropsychiatric Phenomics grants UL1DE019580 and PL1MH083271.

Disclosures There are no conflicts of interest to report.

Open Access This article is distributed under the terms of the Creative Commons Attribution Noncommercial License which permits any noncommercial use, distribution, and reproduction in any medium, provided the original author(s) and source are credited.

\section{References}

Bilder, R. M., Poldrack, R., Parker, D. S., Reise, S. P., Jentsch, J. D., Cannon, T., et al. (2009a). Cognitive phenomics. In S. Wood, N. Allen \& C. Pantelis (Eds.), Handbook of neuropsychology of mental disorders. London: Oxford University Press.

Bilder, R. M., Sabb, F. W., Cannon, T. D., London, E. D., Jentsch, J. D., Parker, D. S., et al. (2009b). Phenomics: the systematic study of phenotypes on a genome-wide scale. Neuroscience.

Bilder, R. M., Sabb, F. W., Parker, D. S., Kalar, D., Chu, W. W., Fox, J., et al. (2009c). Cognitive ontologies for neuropsychiatric phenomics research. Cognitive Neuropsychiatry.

Butcher, L. M., Kennedy, J. K., \& Plomin, R. (2006). Generalist genes and cognitive neuroscience. Current Opinion in Neurobiology, 16 (2), 145-151.

Heaton, R. K., Baade, L. E., \& Johnson, K. L. (1978). Neuropsychological test results associated with psychiatric disorders in adults. Psychological Bulletin, 85, 141-162.

Johnstone, E. C., Crow, T. J., Frith, C. D., Husband, J., \& Kreel, L. (1976). Cerebral ventricular size and cognitive impairment in chronic schizophrenia. Lancet, 2, 924-926.

Sabb, F. W., Bearden, C. E., Glahn, D. C., Parker, D. S., Freimer, N., \& Bilder, R. M. (2008). A collaborative knowledge base for cognitive phenomics. Molecular Psychiatry, 13(4), 350-360.

Sabb, F. W., Burggren, A. C., Higier, R., Fox, J., He, J., Parker, D. S., et al. (2009). Challenges in phenotype definition in the wholegenome era: genetic influences on memory and Intelligence in the healthy population. Neuroscience.

The International Schizophrenia Consortium (2009). Common polygenic variation contributes to risk of schizophrenia and bipolar disorder. Nature, 460, 748-752. 